\title{
Scanning transmission electron microscopy strain measurement from millisecond frames of a direct electron charge coupled device
}

Knut Müller, Henning Ryll, Ivan Ordavo, Sebastian Ihle, Lothar Strüder, Kerstin Volz, Josef Zweck, Heike Soltau, and Andreas Rosenauer

Citation: Appl. Phys. Lett. 101, 212110 (2012); doi: 10.1063/1.4767655

View online: https://doi.org/10.1063/1.4767655

View Table of Contents: http://aip.scitation.org/toc/apl/101/21

Published by the American Institute of Physics

\section{Articles you may be interested in}

Strain mapping at nanometer resolution using advanced nano-beam electron diffraction

Applied Physics Letters 106, 253107 (2015); 10.1063/1.4922994

Two-dimensional strain mapping in semiconductors by nano-beam electron diffraction employing a delay-line detector

Applied Physics Letters 107, 072110 (2015); 10.1063/1.4927837

Local and transient nanoscale strain mapping during in situ deformation

Applied Physics Letters 109, 081906 (2016); 10.1063/1.4961683

Non-spectroscopic composition measurements of $\mathrm{SrTiO}_{3}-\mathrm{La}_{0.7} \mathrm{Sr}_{0.3} \mathrm{MnO}_{3}$ multilayers using scanning convergent beam electron diffraction

Applied Physics Letters 110, 063102 (2017); 10.1063/1.4975932

Strain mapping at the nanoscale using precession electron diffraction in transmission electron microscope with off axis camera

Applied Physics Letters 105, 191906 (2014); 10.1063/1.4901435

An efficient, simple, and precise way to map strain with nanometer resolution in semiconductor devices

Applied Physics Letters 96, 091901 (2010); 10.1063/1.3337090

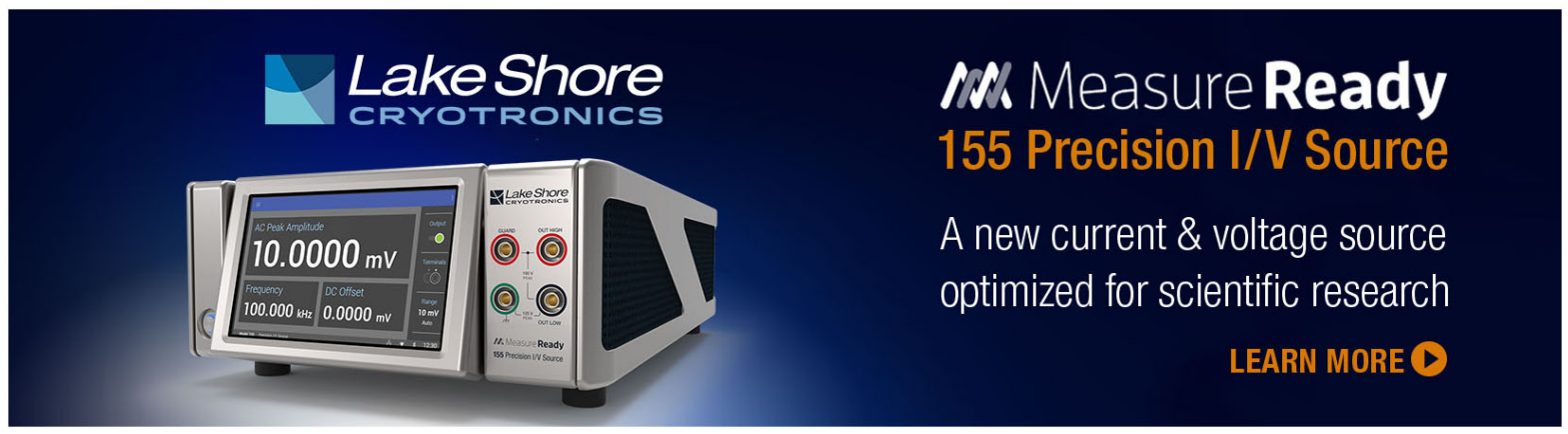




\title{
Scanning transmission electron microscopy strain measurement from millisecond frames of a direct electron charge coupled device
}

\author{
Knut Müller, ${ }^{1, a)}$ Henning Ryll, ${ }^{2}$ Ivan Ordavo, ${ }^{2}$ Sebastian Ihle, ${ }^{2}$ Lothar Strüder, ${ }^{3}$ Kerstin Volz, ${ }^{4}$ \\ Josef Zweck, ${ }^{5}$ Heike Soltau, ${ }^{2}$ and Andreas Rosenauer ${ }^{1}$ \\ ${ }^{1}$ Institut für Festkörperphysik, Universität Bremen, Otto-Hahn-Allee 1, 28359 Bremen, Germany \\ ${ }^{2}$ PNSensor GmbH, Römerstraße 28, 80803 München, Germany \\ ${ }^{3}$ Max-Planck-Institut Halbleiterlabor, Otto-Hahn-Ring 6, 81739 München, Germany \\ ${ }^{4}$ Materials Science Center and Faculty of Physics, Philipps Universität Marburg, Hans-Meerwein-Straße, \\ 35032 Marburg, Germany \\ ${ }^{5}$ Institut für Experimentelle und Angewandte Physik, Universität Regensburg, Universitätsstraße 31, \\ 93040 Regensburg, Germany
}

(Received 1 October 2012; accepted 1 November 2012; published online 21 November 2012)

\begin{abstract}
A high-speed direct electron detection system is introduced to the field of transmission electron microscopy and applied to strain measurements in semiconductor nanostructures. In particular, a focused electron probe with a diameter of $0.5 \mathrm{~nm}$ was scanned over a fourfold quantum layer stack with alternating compressive and tensile strain and diffracted discs have been recorded on a scintillator-free direct electron detector with a frame time of $1 \mathrm{~ms}$. We show that the applied algorithms can accurately detect Bragg beam positions despite a significant point spread each $300 \mathrm{kV}$ electron causes during detection on the scintillator-free camera. For millisecond exposures, we find that strain can be measured with a precision of $1.3 \times 10^{-3}$, enabling, e.g., strain mapping in a $100 \times 100 \mathrm{~nm}^{2}$ region with $0.5 \mathrm{~nm}$ resolution in $40 \mathrm{~s}$. (C) 2012 American Institute of Physics. [http://dx.doi.org/10.1063/1.4767655]
\end{abstract}

Precise quantification of lattice strain with high spatial resolution makes several physical properties of, e.g., semiconductor heterostructures accessible: In computer chip industry, charge carrier mobility in metal oxide field effect transistors (MOSFET) is enhanced ${ }^{1-3}$ by stressors near source and drain. In optoelectronics, local strain is a fingerprint of the local chemical composition and hence plays a key role in understanding spectral properties of light-emitting devices. In the field of transmission electron microscopy (TEM), strain analysis by nano-beam electron diffraction ${ }^{4-6}$ (SANBED) provides the most direct access to lattice strain in the sense that it exploits Bragg beam positions only and is hence independent of beam phases. The latter depends on a variety of parameters of both microscope and specimen and partly makes strain measurement by high-resolution, ${ }^{7-15}$ darkfield, or holographic ${ }^{16,17}$ TEM techniques difficult.

As SANBED methods are based on the analysis of large sets of diffraction patterns taken at raster positions of parallel ${ }^{4,6}$ or convergent ${ }^{5}$ scanning TEM (STEM) probes, their applicability is currently hindered by the limited speed of the acquisition hardware, usually being a scintillator-based charge coupled device (CCD) camera. Whereas a spatial resolution of $0.5 \mathrm{~nm}$ and a strain precision in the range of $10^{-4}$ have been achieved in a recent study by some of the authors, ${ }^{5}$ readout rates of $2 \mathrm{~Hz}$ had to be used to get a proper signal-to-noise ratio of the recorded convergent beam electron diffraction (CBED) patterns.

In this letter, we report on SANBED measurements using a pnCCD detector combined with ultrafast readout hardware based on Refs. 18-20. This sensor is suitable for CBED pattern acquisition with $1 \mathrm{kHz}$ rate, i.e., 500 times

a)mueller@ifp.uni-bremen.de. faster than before. The detector was mounted on a Titan (S)TEM 80/300 (FEI Company, Hillsboro, OR, USA) operated in STEM mode at $300 \mathrm{kV}$ with a semiconvergence angle of $2.6 \mathrm{mrad}$ of the scanning probe. Originating from instrumental research in the field of X-ray detectors for astrophysical and synchrotron applications, the pnCCD chip introduced to TEM here has a size of $264 \times 264$ pixels at $48 \mu \mathrm{m}^{2}$ and is characterised by a high radiation hardness ${ }^{20}$ and a scintillator-free architecture enabling direct electron detection with a detection quantum efficiency close to 1 . A detailed technical description will be presented elsewhere. ${ }^{21}$ A series of 160.000 CBED patterns as depicted in Fig. 1(a) have been recorded with a frame time of $1 \mathrm{~ms}$ while the STEM probe was scanned over a stack of four strained quantum layers buried in GaAs as shown by

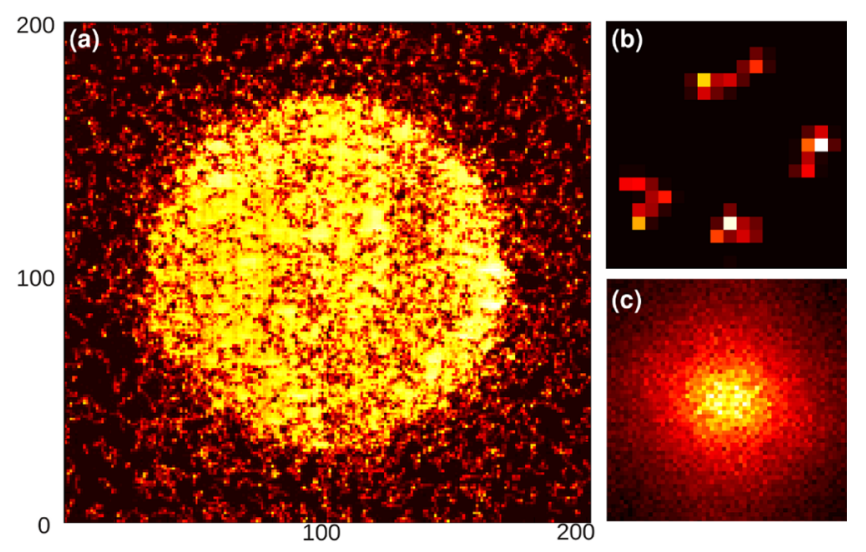

FIG. 1. (a) Experimental pnCCD image of the 400 CBED disc recorded at $300 \mathrm{kV}$ in GaAs with electron beam direction close to [001]. Axes are scaled in pixels. (b) Four single electron detection events taken from a low-dose exposure. (c) Fourier transform of a low-dose exposure exhibiting isotropic point spread. 
the darkfield STEM image in Fig. 2(a). All pnCCD images have been corrected for gain variations among the pixels. The scan path was sampled with 800 scan points at a dwell time of $200 \mathrm{~ms}$ each, allowing for a subsequent integration over up to 200 frames. This sample was particularly chosen because it had been well characterized with respect to strain in Ref. 5, which is why Fig. 2(a) also contains this former strain result to facilitate a comparison. However, Ref. 5 focused on the development of efficient software algorithms for the detection of CBED disc positions, whereas this study addresses the full applicability of SANBED including acquisition.

Raw direct electron images at $300 \mathrm{kV}$ must be analysed with care as becomes obvious from Fig. 1(b), which shows four split events spread over approximately 10 pixels corresponding to four detected electrons. That indeed each of the four traces can be assigned to a single electron event becomes clear from the total counts belonging to each trace which is 3547 in the average, whereas the average difference is 55 counts only. This means that the same amount of energy was deposited in all four events. Quite contrary to the obvious possibility of single-event processing as to a
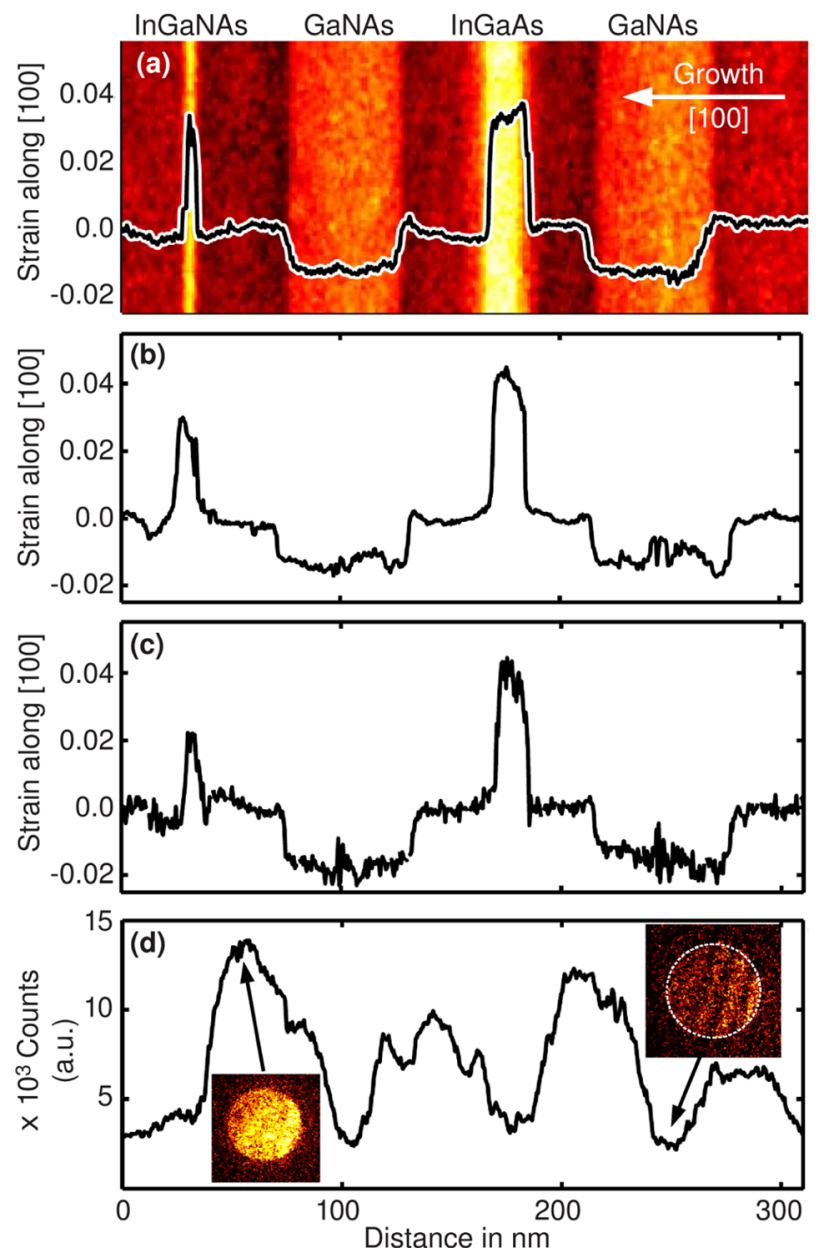

FIG. 2. (a) Dark field STEM image of the investigated sample with a $\mathrm{In}_{x} \mathrm{Ga}_{1-x} \mathrm{~N}_{y} \mathrm{As}_{1-y}$ layer stack buried in GaAs. The strain profile obtained in a former SANBED study ${ }^{5}$ with a conventional CCD and integration times of $500 \mathrm{~ms}$ is superimposed. (b) and (c) Strain profiles measured in the present study using a pnCCD chip with integration times of 200 and $1 \mathrm{~ms}$, respectively. (d) Counts in a millisecond frame versus the scan position. The insets depict examples for high and low excitation of the 400 disc, containing the fitted circle in the latter case. reconstruction of the point of impact with subpixel accuracy, which holds high potential for imaging in conventional TEM, we ignore this point spread here and use electron doses that nearly saturate the detector in the CBED disc area of Fig. 1(a). Consequently, traces of single electrons merge to pile-up events throughout the whole reflection. It is important to note that the counts in the background of the CBED disc are not due to noise but correspond to thermal diffuse scattered electrons or electrons that have excited plasmons.

In the following, we use the radial gradient algorithm ${ }^{5}$ (RGA) to detect positions and radii of CBED discs. As this method basically varies the center of an azimuthal intensity average to maximise the gradient near the disc boundary, the point spread is required to be isotropic. This was checked in Fig. 1(c), which shows the power spectrum of a raw $300 \mathrm{kV}$ pnCCD image without pile-up events taken under homogeneous low-dose illumination. Since the spectrum is rotationally symmetric, we conclude that no preferred direction exists for the split events, meaning that also the CBED discs broaden isotropically.

According to Bragg's equation, ${ }^{22}$ the position of the 400 disc in Fig. 1(a) varies with the (400) lattice plane spacing in the illuminated specimen volume. As the semiconvergence angle of the STEM probe is known, these shifts can be converted to angular changes $\Delta \theta$ using the radius in pixels obtained by the RGA. Finally, the strain in [100] direction is given by

$$
\varepsilon_{[100]}=\frac{\sin \theta_{400}^{B}}{\sin \left(\theta_{400}^{B}+\Delta \theta / 2\right)}-1
$$

with $\theta_{400}^{B}$ the Bragg angle of the 400 reflection in GaAs.

A resulting strain profile calculated by means of Eq. (1) from CBED patterns with an integration time of $200 \mathrm{~ms}$ (sum of 200 frames) is shown in Fig. 2(b). The alternating sequence of compressive and tensile strain is well resolved and agrees with the former (Ref. 5) result depicted in Fig. 2(a). Except for small differences on the left of the InGaNAs layer both profiles (a) and (b) also match quantitatively. Note that Fig. 2(a) stems from a different position on the specimen which might be the reason for marginal differences inside the GaNAs layers. As to the precisions of the strain measurements, we find nearly identical values of 7.3 and $7.0 \times 10^{-4}$ from the standard deviation of 30 strain measurements at the right end of the profiles in Figs. 2(a) and 2(b), respectively. Although the result of Fig. 2(b) effectively corresponds to a frame time of $200 \mathrm{~ms}$ which did not yet speed up the acquisition in comparison to $500 \mathrm{~ms}$ in Fig. 2(a), this is already a remarkable result for two reasons: First, it shows that ignoring the point spread which broadens the CBED disc edge significantly is not only justified with respect to the RGA but it also yields an even slightly better precision than in Ref. 5 . Second, no energy filtering or specimen cooling has been applied in the current study, whereas the strain profile in Fig. 2(a) stems from zero-loss energy-filtered CBED patterns at $99 \mathrm{~K}$. On the other hand, the disc radius in Fig. 1(b) is approximately 125 pixels which is $30 \%$ more than in Fig. 1(a), which enhances the precision. As a first result, we, therefore, find that SANBED combined with a direct electron 
pnCCD chip principally allows for strain measurements with contemporary ${ }^{4-6}$ precisions.

Since our aim is high-speed strain map acquisition, we investigated the behaviour of the pnCCD strain profiles when the frame time is decreased to $10,5,2$, and $1 \mathrm{~ms}$ of which the last one is plotted in Fig. 2(c). Again this profile accurately reproduces the measurements of Figs. 2(a) and 2(b), albeit with a different precision of $1.3 \times 10^{-3}$. On the one hand, this means a decrease of precision by a factor of 1.8 , but on the other hand one must keep in mind that the raw signal is a factor of 200 weaker and the acquisition 200 times faster, too. In fact, this precision is still sufficient for most applications as it translates to a precision of, e.g., $\sigma_{x}=0.01$ for the In composition measurement in $\mathrm{In}_{x} \mathrm{Ga}_{1-x}$ As with $x=0.28$, which corresponds to the InGaAs quantum well in Fig. 2(a).

Furthermore, it is instructive to look at Fig. 2(d) which shows the intensity in the patch around the 400 disc for each scan position together with two sample images, one for high and one for low excitation of the 400 beam. Despite the strongly varying intensity, the graph in Fig. 2(c) does not exhibit a strict correlation to Fig. 2(d) as to the precision: Although fluctuations in the GaNAs layers in Fig. 2(c) occur at intensity minima, not each minimum results in lower precision as becomes obvious near the InGaAs layer as well as at the left and right ends of the strain profile. As the circle in the right inset shows, the RGA still yields reasonable results even if the intended intensity distribution of Fig. 1(a) is not achieved throughout the whole scan. Whereas an azimuthal intensity average for the bright disc drops by a factor of 8 at the disc edge, this drop is at least 4 for the right inset in Fig. 2(d), which one might not guess for this seemingly noisy image. Consequently, faster readout rates should yield competitive strain precisions in future experiments. This also means that much weaker reflections, such as 800 , can be used for strain evaluation which is preferable due to a linear dependence of shifts $\Delta \theta$ on reflection order in Eq. (1) for high energy electron diffraction. To conclude, we find that the present detector allows for accurate strain measurements even from millisecond exposures with a precision of $1.3 \times 10^{-3}$.

In Table I, the interconnection between strain precision and integration time is demonstrated in more detail where we additionally analysed our data for different integration times in the same way as in Figs. 2(b) and 2(c). On the one hand, the expected trend of lower precisions for decreasing integration times is visible. On the other hand, we can temporarily leave this methodically motivated study in which we oversampled the data acquisition by a factor of 200 and consider an experiment where both dwell time of the STEM probe and pnCCD exposure of $1 \mathrm{~ms}$ are matching. Assuming the current resolution limit of $0.5 \mathrm{~nm}$ for SANBED strain mapping $^{5}$ and a typical application, e.g., characterization of

TABLE I. Interconnection between frame time for a single CBED image and precision of the strain measurement.

\begin{tabular}{lccccc}
\hline \hline Frame time $[\mathrm{ms}]$ & 1 & 2 & 5 & 10 & 200 \\
Strain precision $\left[1 \times 10^{-4}\right]$ & 13 & 12 & 9.5 & 9.2 & 7.0 \\
\hline \hline
\end{tabular}

a $100 \times 100 \mathrm{~nm}^{2}$ large MOSFET region, acquisition of a strain map would take 40 and $80 \mathrm{~s}$ for pnCCD readout rates of 1 and $0.5 \mathrm{kHz}$, respectively, which are typical durations also for high-resolution dark field STEM records. In contrast, acquisitions with the conventional setup ${ }^{5}$ would take more than $5.5 \mathrm{~h}$, which demonstrates the capability of the direct electron CCD used here.

In conclusion, we have shown that the combination of contemporary TEM with the latest generation of electron detectors and readout hardware enables strain mapping with a precision of $1.3 \times 10^{-3}$ with millisecond dwell time in STEM mode. This extends the scope of application for direct electron detectors, whose excellent detection quantum efficiency and signal-to-noise ratio have as yet enhanced nearly uniquely low-dose applications as used for biological imaging. ${ }^{23-27}$ In this study, we used $300 \mathrm{keV}$ electrons as the microscope performs best at this energy as to STEM probe formation. However, it can be expected that lowering the TEM acceleration voltage to less than $100 \mathrm{kV}$ will lead to a strong improvement of the camera's point spread function, from which both SANBED and conventional TEM applications will profit.

${ }^{1}$ A. Lochtefeld and D. Antoniadis, IEEE Electron Device Lett. 22, 591 (2001).

${ }^{2}$ L. Smith, V. Moroz, G. Eneman, P. Verheyen, F. Nouri, L. Washington, M. Jurczak, O. Penzin, D. Pramanik, and K. De Meyer, IEEE Electron Device Lett. 26, 652 (2005).

${ }^{3}$ C. D. Krzeminski, J. Vac. Sci. Technol. B 30, 022203 (2012).

${ }^{4}$ A. Béché, J. L. Rouvière, L. Clément, and J. M. Hartmann, Appl. Phys. Lett. 95, 123114 (2009).

${ }^{5}$ K. Müller, A. Rosenauer, M. Schowalter, J. Zweck, R. Fritz, and K. Volz, Microsc. Microanal. 18, 995 (2012).

${ }^{6}$ A. Béché, L. Clément, and J.-L. Rouvière, J. Phys.: Conf. Ser. 209, 012063 (2010).

${ }^{7}$ R. Bierwolf, M. Hohenstein, F. Phillipp, O. Brandt, G. Crook, and K. Ploog, Ultramicroscopy 49, 273 (1993).

${ }^{8}$ A. Rosenauer, U. Fischer, D. Gerthsen, and A. Förster, Ultramicroscopy 72, 121 (1998).

${ }^{9}$ A. Rosenauer, D. Gerthsen, and V. Potin, Phys. Status Solidi A 203, 176 (2006).

${ }^{10}$ K. Müller, M. Schowalter, A. Rosenauer, D. Hu, D. M. Schaadt, M. Hetterich, P. Gilet, O. Rubel, R. Fritz, and K. Volz, Phys. Rev. B 84, 045316 (2011).

${ }^{11}$ K. Tillmann, M. Lentzen, and R. Rosenfeld, Ultramicroscopy 83, 111 (2000).

${ }^{12}$ W. Yu and W. Mader, Ultramicroscopy 110, 411 (2010).

${ }^{13}$ F. Hüe, M. Hýtch, H. Bender, F. Houdellier, and A. Claverie, Phys. Rev. Lett. 100, 156602 (2008).

${ }^{14}$ F. Hüe, M. Hýtch, F. Houdellier, H. Bender, and A. Claverie, Appl. Phys. Lett. 95, 073103 (2009).

${ }^{15}$ M. J. Hýtch, J.-L. Putaux, and J.-M. Pénisson, Nature 423, 270 (2003).

${ }^{16}$ M. Hýtch, F. Houdellier, F. Hüe, and E. Snoeck, Nature 453, 1086 (2008).

${ }^{17}$ C. T. Koch, V. B. Özdöl, and P. A. van Aken, Appl. Phys. Lett. 96, 091901 (2010).

${ }^{18}$ L. Strüder, H. Bräuninger, U. Briel, R. Hartmann, G. Hartner, D. Hauff, N. Krause, B. Maier, N. Meidinger, E. Pfeffermann, M. Popp, C. Reppin, R. Richter, D. Stötter, J. Trümper, U. Weber, P. Holl, J. Kemmer, H. Soltau, A. Viehl, and C. Zanthier, Rev. Sci. Instrum. 68, 4271 (1997).

${ }^{19}$ R. Hartmann, W. Buttler, H. Gorke, S. Herrmann, P. Holl, N. Meidinger, H. Soltau, and L. Strüder, Nucl. Instrum. Methods Phys. Res. A 568, 118 (2006).

${ }^{20}$ I. Ordavo, S. Ihle, V. Arkadiev, O. Scharf, H. Soltau, A. Bjeoumikhov, S. Bjeoumikhova, G. Buzanich, R. Gubzhokov, A. Günther, R. Hartmann, P. Holl, N. Kimmel, M. Kühbacher, M. Lang, N. Langhoff, A. Liebel, M. Radtke, U. Reinholz, H. Riesemeier, G. Schaller, F. Schopper, L. Strüder, C. Thamm, and R. Wedell, Nucl. Instrum. Methods Phys. Res. A 654, 250 (2011). 
${ }^{21}$ H. Ryll, I. Ordavo, K. Müller, S. Ihle, H. Soltau, and L. Strüder, "A pnCCD-based, fast direct single electron imaging camera for transmission electron microscopy," Microsc. Microanal. (unpublished).

${ }^{22}$ W. H. Bragg and W. L. Bragg, Proc. R. Soc. London, Ser. A 88, 428 (1913).

${ }^{23}$ B. E. Bammes, R. H. Rochat, J. Jakana, D.-H. Chen, and W. Chiu, J. Struct. Biol. 177, 589 (2012).

${ }^{24}$ A. F. Brilot, J. Z. Chen, A. Cheng, J. Pan, S. C. Harrison, C. S. Potter, B. Carragher, R. Henderson, and N. Grigorieff, J. Struct. Biol. 177, 630 (2012).
${ }^{25}$ A.-C. Milazzo, A. Cheng, A. Moeller, D. Lyumkis, E. Jacovetty, J. Polukas, M. H. Ellisman, N.-H. Xuong, B. Carragher, and C. S. Potter, J. Struct. Biol. 176, 404 (2011).

${ }^{26}$ A.-C. Milazzo, G. Moldovan, J. Lanman, L. Jin, J. C. Bouwer, S. Klienfelder, S. T. Peltier, M. H. Ellisman, A. I. Kirkland, and N.-H. Xuong, Ultramicroscopy 110, 741 (2010).

${ }^{27}$ L. Jin, A.-C. Milazzo, S. Kleinfelder, S. Li, P. Leblanc, F. Duttweiler, J. C. Bouwer, S. T. Peltier, M. H. Ellisman, and N.-H. Xuong, J. Struct. Biol. 161, 352 (2008). 